BULL. AUSTRAL. MATH. SOC.

VOL. $20(1979), 207-210$.

\title{
Bitangents of plane quartics
}

\section{J.P. Glass}

The topic dealt with in this paper arose out of a study of theta functions and the moduli of curves. It concerns itself with configurations of lines in the plane and when they can be bitangents to a quartic. The techniques used are classical.

\section{Bitangents}

In the paper [6] we showed that points of $V_{3}$ with no zero coordinates belong to the strong closure of the locus of non-hyperelliptic curves of genus three. Our aim here is to prove that each such point of $V_{3}$ always corresponds to a non-hyperelliptic curve. The method of associating a curve to such a point of $V_{3}$ was well defined in [6], but it was not shown that the curve is necessarily non-singular.

We recall that the associated plane quartic had been studied by Riemann and that he had written down formulae for the 28 bitangents in the case that the curve is non-singular. Cayley, in [4], p. 74, showed that in the reverse direction these 28 lines are always bitangents to that quartic curve. In order to prove that the quartic is non-singular it will suffice to demonstrate that these 28 bitangents are distinct, and this shall be our approach.

We begin with a point of $V_{3}$ with no zero coordinates and associate to it the moduli $\alpha_{i}, \alpha_{i}^{\prime}, \alpha_{i}^{\prime \prime}$. Then take the seven lines

Received 2 February 1979. The author has received invaluable assistance from Dr Patrick Du Val, and from his $\mathrm{PhD}$ supervisor, Sir Peter Swinnerton-Dyer, in the first and second sections, respectively. He would like to express his gratitude to both of them. 


$$
\begin{gathered}
t_{1}=0, t_{2}=0, t_{3}=0, t_{1}+t_{2}+t_{3}=0, \\
\alpha_{1} t_{1}+\alpha_{2} t_{2}+\alpha_{3} t_{3}=0, \alpha_{1}^{\prime} t_{1}+\alpha_{2}^{\prime} t_{2}+\alpha_{3}^{\prime} t_{3}=0, \alpha_{1}^{\prime \prime} t_{1}+\alpha_{2}^{\prime \prime} t_{2}+\alpha_{3}^{\prime \prime} t_{3}=0 .
\end{gathered}
$$

It is proved in [5], p. 4.25, that these seven lines are distinct. Dualising, look at the seven points $0_{1}, \ldots, 0_{7}$ dual to these lines. Then the projective model of the net of cubics through these seven points will give a double plane (see [8], Chapter VII). Call the jacobian curve of the net $J^{6}$ and $B^{4}$ its quartic image. The construction of Aronhold (see [1] or [7]) determines the quartic we are investigating, which we shall call $Q^{4}$. It can be shown that $Q^{4}$ and $B^{4}$ are both birationally equivalent to $J^{6}$ and hence are projectively equivalent.

Therefore we only need to show that the 28 bitangents of $B^{4}$ are distinct. The bitangents of $B^{4}$ are the images of cubics in the plane containing $J^{6}$. These cubics are either of the form

(a) the line $0_{i} 0_{j}$ and the conic through the remaining 5 points, or

(b) the unique cubic of the net with a double point at $0_{i}$. We shall call these $(i j)$ and $(i 8)$, respectively.

Using this labelling of the bitangents, there are several general cases to look at. Firstly, if either $(12)=(34)$ or $(56)=(78)$, we can conclude that $0_{5}, 0_{6}, 0_{7}$ are collinear. This will contradict the fact that the original point $P$ of $V_{3}$ has no zero coordinates. Secondly, if $(67)=(78)$ we conclude that $0_{1}, 0_{2}, \ldots, 0_{6}$ lie on a conic. This contradicts the formulae in Weber [9] which express the moduli $\alpha_{i}^{\prime \prime}$ in terms of the $\alpha, \alpha^{\prime}$. Finally, if $(16)=(17)$ or $(68)=(78)$, we conclude that ${ }_{6}{ }_{6} 0_{7}$ but, as stated above, our seven points are distinct. This completes the proof that the point $P$ corresponds to a non-hyperelliptic curve. 


\section{An alternative approach}

The proof that the associated curve is non-singular can be approached in another way. The Plücker formulae show that if a quartic has one ordinary double point, then there are only 16 proper bitangents. The other 12 coincide in six pairs as lines passing through the double point and touching the curve elsewhere. If there are more, or more complicated, singularities then there are further coincidences. In this section we will prove that the quartic generated by a point of $V_{3}$ (with no zero coordinates) can not even have a single ordinary double point. We will restrict the exposition to a mere outline of the ideas involved.

We use the well-known correspondence between the plane quartic with its 28 bitangents and the cubic surface with its 27 lines. In addition, some results of Cayley will be used (see [2], p. 372; [3], p. 316). Fixing a bitangent $I_{0}$, its stabiliser in $S_{6}(2)$ is the same as the group of the 27 lines. Moreover, two lines on the surface intersect if and only if the sum of their characteristics with that of $I_{0}$ is odd.

When a cubic surface acquires a node in a degeneration, 12 of the 27 lines coincide in pairs. Cayley ([2], [3]) has proved that these 12 lines form a double-sixer. It can also be proved that if $c$ is any even characteristic, then two lines $I_{i}, l_{j}$ are a pair of corresponding lines in the double-sixer belonging to $c$ if and only if $\operatorname{char} l_{i}+\operatorname{char} I_{j}=c-\operatorname{char} 1_{0}$. This implies that the six pairs of degenerate bitangents passing through the ordinary double point have the property that for each pair, the sum of characteristics of the lines is the same. Hence we need to take each non-zero characteristic (even or odd) and write it in six ways as a sum of two odd characteristics (this always being possible in precisely six ways). In each case we need only take one pair of lines and check that the lines are distinct. Many cases drop out by symmetry and, in the end, the desired result can be achieved without tao much hard work. 


\section{References}

[1] [S.H.] Aronhold, "Über den gegenseitigen Zusammenhang der 28 Doppeltangenten einer allgemeinen Curve 4ten Grades", Monatsberichte K. Preuss. Akad. Wiss. Berlin, 1864, 499-523 . (1865).

[2] Arthur Cayley, The collected mathematical papers of Arthur Cayley, Volume VI (Cambridge University Press, Cambridge, 1893).

[3] Arthur Cayley, The collected mathematical papers of Arthur Cayley, Volume VII (Cambridge University Press, Cambridge, 1894).

[4] Arthur Cayley, The collected mathematical papers of Arthur Cayley, Volume XII (Cambridge University Press, Cambridge, 1897).

[5] Jonathan Paul Glass, "Theta constants of genus three" (PhD thesis, University of Cambridge, Cambridge, 1977).

[6] J.P. Glass, "Theta constants of genus three", Compositio Math. (to appear).

[7] George Salmon, A treatise on the higher plone curves, intended as a sequel to a treatise of conic sections, second edition (Hodges, Foster and Co., Dublin, 1873).

[8] J.G. Semple and L. Roth, Introduction to algebraic geometry (Clarendon Press, Oxford, 1949).

[9] H. Weber, Theorie der Abel'schen Functionen vom Geschlecht 3 (Reimer, Berlin, 1876). [See Jahrbuch über die Fortschritte der Mathematik 8 (1876), 293-299.]

Department of Pure Mathematics,

University of Sydney,

Sydney,

New South Wales.

Present Address:

School of Mathematics,

University of New South Wales,

Kensington,

New South Wales. 